\title{
Intravenous Administration of Toll-Like Receptor Inhibitory Peptide 1 is Effective for the Treatment of Systemic Lupus Erythematosus in a Mus musculus Model
}

\author{
Wook-Young Baek, Ph.D. ${ }^{1,2} *$, Sung-Min Lee, MSc ${ }^{1}$, Sang-Won Lee, MSc ${ }^{1}$, In-Ok Son, MSc ${ }^{1}$, Sangdun Choi, Ph.D. ${ }^{2}$, \\ Chang-Hee Suh, M.D., Ph.D. ${ }^{1,2}$ \\ ${ }^{1}$ Department of Rheumatology, Ajou University School of Medicine, ${ }^{2}$ Department of Molecular Science and Technology, Ajou University, \\ Suwon, Korea
}

\begin{abstract}
Objective. Systemic lupus erythematosus (SLE) is a common chronic autoimmune inflammatory disease. According to recent studies, signaling through Toll-like receptor (TLR) protein, which promotes the production of inflammatory cytokines, leads to the development of SLE. TLR-inhibitory peptide 1 (TIP1) has been newly identified for the treatment of autoimmune diseases. Methods. The effect of TIP1 was analyzed in an SLE mouse model (MRL/lpr). The mice in the control treatment group ( $\mathrm{n}=5$ ) were administered an intravenous injection of phosphate-buffered saline twice weekly, whereas the mice in the TIP1 treatment group $(n=6)$ were administered an intravenous injection of TIP1 $(1 \mathrm{nmol} / \mathrm{g})$ twice weekly. MRL/mpj mice $(n=5)$ were selected as normal controls. The mice were injected for 4 weeks between 14 and 18 weeks of age, followed by assays of their spleen, kidneys, lymph nodes, serum, and urine. Results. The antinuclear antibody and inflammatory cytokine (interferon- $\alpha$ ) in the serum as well as levels of albumin in the urine of the mice in the TIP1 treatment group had decreased when compared to those of mice in the control treatment group. Kidney inflammation in mice in the TIP1 treatment group was alleviated. The mRNA expression levels of TLR7- or TLR9-related downstream signaling molecules also decreased in all organs of the mice in the TIP1 treatment group. Conclusion. Intravenous treatment with TIP1 reduces symptoms and markers of inflammation in MRL/lpr mice. Hence, TIP1 is a promising medication for the treatment of SLE. (J Rheum Dis 2021;28:133-142)
\end{abstract}

Key Words. Systemic lupus erythematosus, Inflammation, MRL Ipr mice, Toll-like receptor

\section{INTRODUCTION}

Being an autoimmune disease, systemic lupus erythematosus (SLE), commonly called lupus, causes damage to body parts, including skin, joints, blood, lymph nodes, kidneys, and spleen, owing to excessive production of inflammatory cytokines [1]. SLE also induces skin inflammation, which leads to skin rashes. Further, invasion of cytokines in the kidneys causes lupus nephritis, followed by the development of nephrotic syndrome, and deteriorates kidney function, leading to renal failure $[2,3]$. Furthermore, autoantibodies including antinuclear antibodies (ANAs) and anti-dsDNA antibodies are produced, along with the development of lymphadenitis that results in hyperplasia of the lymph nodes [4].

Causes of SLE have not yet been clearly identified, but abnormal immunological reactions are commonly assumed to cause SLE. It has been suggested that immunological reactions are associated with genetic, environmental, and hormonal factors [5]. Considering SLE is most common in young women of reproductive age (in their 20s or 30s) and its incidence decreases with age, SLE may also be related to female hormones [6,7]. It has also been reported that several types of immune cells, such as

\footnotetext{
Received : January 11, 2021, Revised : February 23, 2021, Accepted : March 1, 2021

Corresponding to : Chang-Hee Suh $\mathbb{D}$ http://orcid.org/0000-0001-6156-393X

Department of Rheumatology, Ajou University School of Medicine, 164 Worldcup-ro, Yeongtong-gu, Suwon 16499, Korea. E-mail : chsuh@ajou.ac.kr

*These authors contributed equally to this work.
}

Copyright (c) 2021 by The Korean College of Rheumatology.

This is an Open Access article, which permits unrestricted non-commerical use, distribution, and reproduction in any medium, provided the original work is properly cited. 
macrophages and dendritic cells, are associated with an overactive phenotype and function [8-10]. Recent studies have demonstrated that abnormal control of the Toll-like receptor (TLR) pathway involved in the innate immune system, the TLR7 or TLR9 pathway, may play a crucial role in the activation of macrophages and dendritic cells [11-13]. In addition, abnormal control of the TLR pathway may also be involved in the development and progression of SLE as well as the activation of immune cells, considering that the increased production of TLR7 leads to higher level of disease activity [14-16].

Kwon et al. [17] traced a Toll/interleukin-1 receptor (TIR) domain functioning as a TLR signaling adaptor and discovered a peptide controlling TLR activation signals. It was reported that the TLR-inhibiting peptide (TIP) 1, derived from the TIR domain, specifically penetrates the cell membrane and blocks the downstream signaling cascade through the myeloid differentiation primary response gene 88 (MyD88). In addition, it has been found that TIP1 effectively inhibits cytokine secretion primarily induced by the signaling pathways of TLR4, TLR7, TLR8, and TLR9 [17]. In our study, TIP1 was intravenously injected in mouse models of SLE (MRL/lpr), and their skin lesions, lymph nodes, spleen, and kidneys were analyzed to determine whether their SLE symptoms or signs could be alleviated. In addition, we examined TLR7- or TLR9-related downstream signaling molecules, including MyD88, interleukin-1 receptor-associated kinase 4 (IRAK4), tumor necrosis factor receptor-associated factor 6 (TRAF6), interferon regulatory factor 7 (IRF7), and inflammatory cytokines such as interferon- $\alpha$ (IFN- $\alpha$ ).

\section{MATERIALS AND METHODS}

\section{Peptides}

TIP1 peptides were constructed by solid-phase synthesis (PEPTRON, Daejeon, Korea) and the purity above $95 \%$ of each peptides was confirmed by high-performance liquid chromatography.

\section{Animals}

All animal procedures were reviewed and approved by the animal ethics committee of our institute (approval no. 2018-0029). Mice were maintained in accordance with the guidelines of the animal facility at our institution in pathogen-free conditions to acclimate them for 1 week after purchase. Female MRL/mpjmice $(\mathrm{n}=5)$ were selected as normal controls, and female MRL/lpr mice were se- lected for the control treatment group $(\mathrm{n}=5)$ and TIP1 treatment group $(\mathrm{n}=6)$. MRL/mpjmice had the same genetic background as MRL/Ipr mice. All mice were purchased from The Jackson Laboratory (Bar Harbor, ME, USA). The mice were injected from 14 to 18 weeks of age, for a total of 4 weeks. Further, 1X phosphate-buffered saline was injected twice weekly in the mice in the normal control and MRL/lpr control treatment group. TIP1 (1 $\mathrm{nmol} / \mathrm{g}$ ) was injected twice weekly in the mice in the TIP1 treatment group. All mice were weighed weekly for 4 weeks. After the injection period, 18-week-old mice were anesthetized with respiratory anesthesia using isoflurane. Blood and urine samples were collected immediately before euthanasia, and spleen, lymph node, and kidney samples were obtained immediately after euthanasia. Blood was immediately collected in a serum separation tube (BD Microtainer SST; BD Biosciences, San Jose, CA, USA) and centrifuged at $3,393 \mathrm{~g}$ for 30 minutes at $4^{\circ} \mathrm{C}$ to separate the serum, which was then stored at $-70^{\circ} \mathrm{C}$. Each organ was cut into $5 \mathrm{~mm}$ pieces, stored in an RNA Stabilization Reagent (RNAlater, Qiagen, Hilden, Germany), and maintained at $-20^{\circ} \mathrm{C}$.

\section{Histologic assessment of kidney}

Fragments of the kidney from MRL/lpr mice were prepared immediately after euthanasia. After fixation in $4 \%$ paraformaldehyde for 24 hours, the samples were dehydrated in ethanol, embedded in Paraplast (SigmaAldrich, St. Louis, MO, USA). And then, $2 \sim 3 \mu \mathrm{m} \mathrm{sec-}$ tions were then stained with hematoxylin and eosin (H\&E) and Periodic Acid Schiff (PAS). In addition, stained slides were read and interpreted in a blinded manner and evaluated as histopathological nephritis index. The nephritis index indicated the score of the kidney section on a scale of $0 \sim 4$ for the intensity of glomerular inflammatory cell infiltration and the presence of glomerular necrosis. (Where glomerular inflammation is 0 , normal; 1, 2, 3, and 4 each represent few inflammatory cells, moderate inflammation, severe inflammation, and severe abnormalities, respectively) [18].

\section{Real-time polymerase chain reaction assay}

The mRNAs of the spleen, kidney, and lymph nodes were extracted using the FavorPrep Tissue Total RNA Purification Mini Kit (Favorgen Biotech Corporation, Pingtung, Taiwan) according to the manufacturer's protocol. The mRNA isolated from each tissue was synthesized using the AMPIGENE cDNA Synthesis Kit 
(Enzo Life Sciences, Inc., Farmingdale, NY, USA) according to the manufacturer's instructions. The synthesized cDNA was subjected to a real-time polymerase reaction using AMPIGENE qPCR Green Mix (Enzo Life Sciences, Inc.). Real-time PCR was performed 35 times and included initialization at $95^{\circ} \mathrm{C}$ for 5 minutes, denaturation at $95^{\circ} \mathrm{C}$ for 30 seconds, annealing at $60^{\circ} \mathrm{C}$ for 1 minute, and extension at $72^{\circ} \mathrm{C}$ for 30 seconds. mRNA expression levels of TLR7, TLR9, and downstream signaling proteins were measured.

\section{Western blotting}

For Western blotting, the total protein was isolated using RIPA Buffer (Intron Biotechnology, Seoul, Korea), and each tissue was quantified according to the manufacturer's protocol using the Pierce BCA Protein Assay Kit (Thermo Fisher Scientific, Rockford, IL, USA). After euthanizing the mice, the extracted spleen, kidney, and lymph node tissue proteins were separated by electrophoresis and transferred to a polyvinylidene fluoride membrane. Then, the membrane was blocked with 5\% bovine serum albumin (MP Biomedicals, Irvine, CA, USA). Further, the membrane was stained with $\beta$-actin antibody (A300-491A; Bethyl Laboratories, Montgomery, TX, USA), TLR7 antibody (\#2633; Cell Signaling Technology, Inc., Danvers, MA, USA), TLR9 antibody (LS-B688; LS Bio, Seattle, WA, USA), MyD88 antibody (LS-C295471, LS Bio), IRAK4 antibody (LS-C334563, LS Bio), TRAF6 antibody (LS-C331172, LS Bio), and IRF7 antibody (LS-B577, LS Bio). Western blot bands were analyzed using Image J (Bethesda, MD, USA) software bundled with 64-bit Java 1.8.0_112. The expression level of a specific protein was compared with the $\beta$-actin value and then statistically analyzed.
A

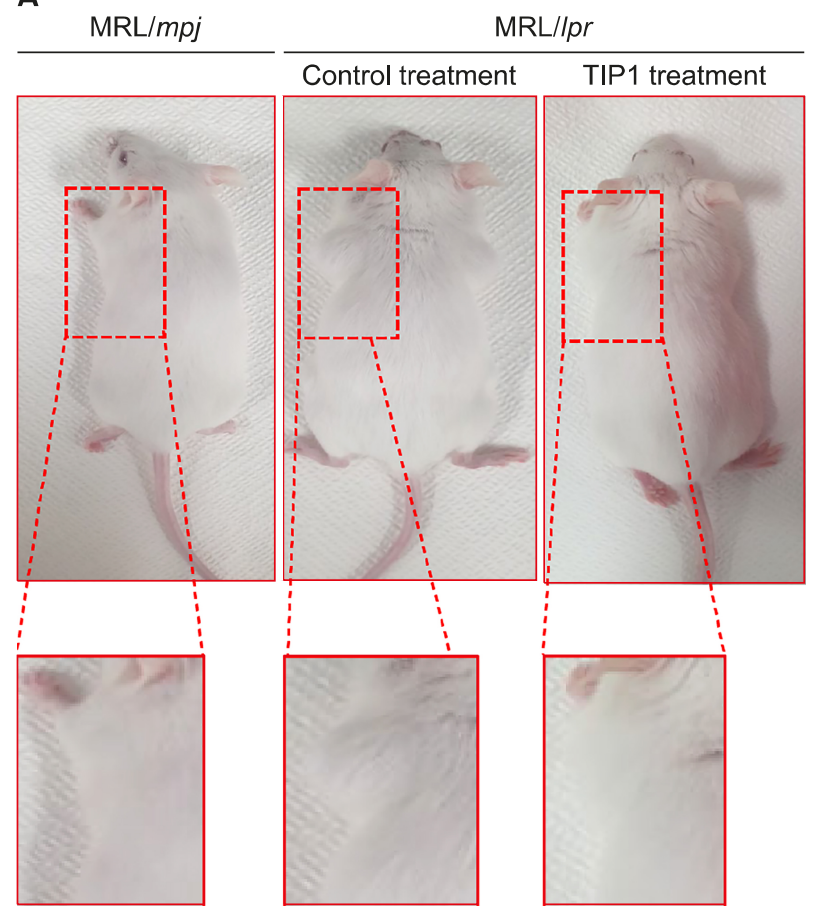

B

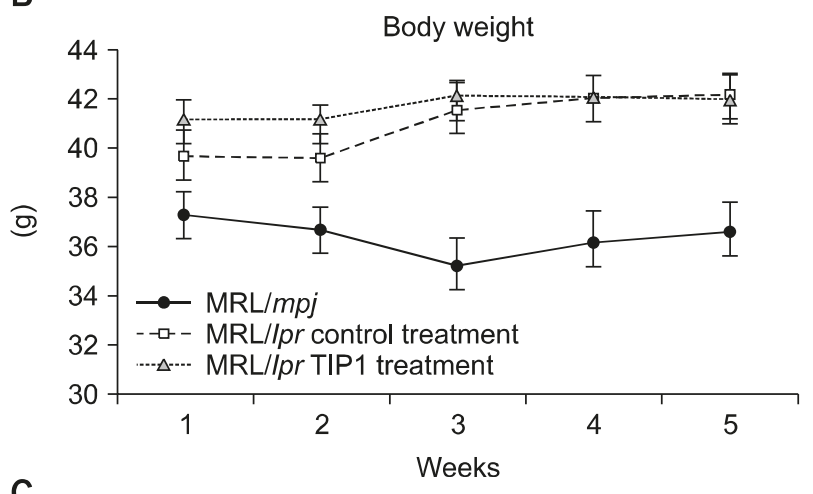

C $\mathrm{MRL} / m p j=\frac{\mathrm{MRL} / / p r}{\text { Control treatment }}$ In1 MPY MPN

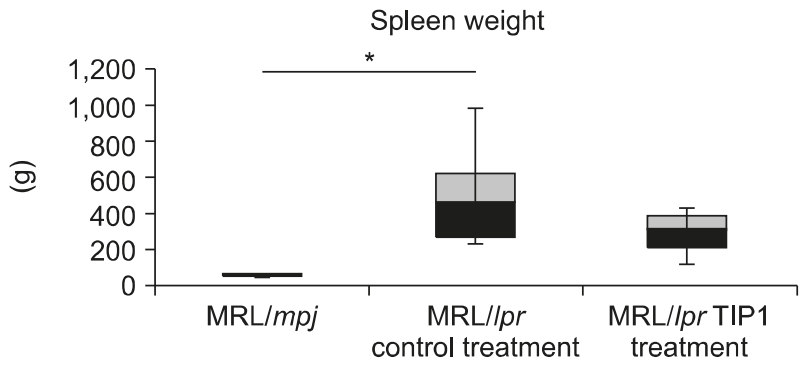

Figure 1. Effect of treatment with Toll-like receptor inhibitory peptide 1 (TIP1) in an SLE mouse model. (A) Whole body images of MRL/lpr mice and age- and sex-matched controls (MRL/mpj) are shown. These images show the (B) change in the weight of the mice during the 4-week injection period and (C) effect of TIP1 treatment on spleen hypertrophy in MRL/lpr mice. SLE: systemic lupus erythematosus. ${ }^{*} p<0.05$. 


\section{Enzyme-linked immunosorbent assay (ELISA)}

For ELISA, Mouse Albumin ELISA Kit (Alpco Diagnostics, Salem, NH, USA), ANA ELISA Kit (MyBioSource, San Diego, CA, USA), anti-dsDNA ELISA Kit (Mybiosource), and Verikine-HS Mouse IFN- $\alpha$ ELISA Kit (PBL Assay Science, Piscataway, NJ, USA) were used according to the manufacturer's instructions. Albumin was identified using mouse urine, and mouse serum was used for tests with ANA, anti-dsDNA antibody, and IFN- $\alpha$.

\section{Statistical analysis}

To confirm the significance of the difference in protein expression levels between the control treatment and TIP1 treatment groups, the results of the tests were verified by the Mann-Whitney U-test using the IBM Statistical Package for the Social Sciences (SPSS) Statistics 25.0 (IBM Corporation, Armonk, NY, USA) program. When the p-value was less than 0.05 , the difference was considered statistically significant.

\section{RESULTS}

\section{Body weight change and organomegaly due to TIP1 treatment in mice}

In this study, we analyzed the phenotype of the entire mouse body for the first time. The mice in the control treatment group showed lymph node enlargement, which is the main symptom of SLE. However, TIP1 treatment of $\mathrm{MRL} /$ Ipr mice resulted in reduction of the lymph node enlargement (Figure 1A). Because it is known that body weight may increase owing to organomegaly when symptoms of SLE appear [4], we investigated the changes in the body weight of mice following treatment with TIP1. All mice were weighed once a week, starting from immediately before injection to just before euthanasia. The average body weight of mice in the control treatment group showed a tendency to increase continuously, but the average body weight of mice in the TIP1 treatment group tended to stabilize (Figure 1B). The difference in the average weight of the spleen between mice in the two groups was not statistically significant, but the spleen weight decreased slightly in the mice in the TIP1 treatment group. However, the length of the spleen did not change in the mice in either group (Figure 1C). The average kidney weight of the mice in the TIP1 treatment group reduced to a small extent when compared to that of the mice in the control treatment group, but the difference was not statistically significant (data not shown). Further, the lymph nodes of mice in the control treatment group were enlarged, but the enlargement was reduced marginally in mice in the TIP1 treatment group.

\section{Kidney tissue and inflammation after TIP1 treatment}

SLE usually invades the kidney tissue and causes inflammation, resulting in lupus nephritis. Lupus nephritis induces inflammation of blood vessels that filter waste products from the kidneys, causing mesangial cells to proliferate. Further, the basement membrane cells outside the glomerular blood vessels invade, and plasma pro-
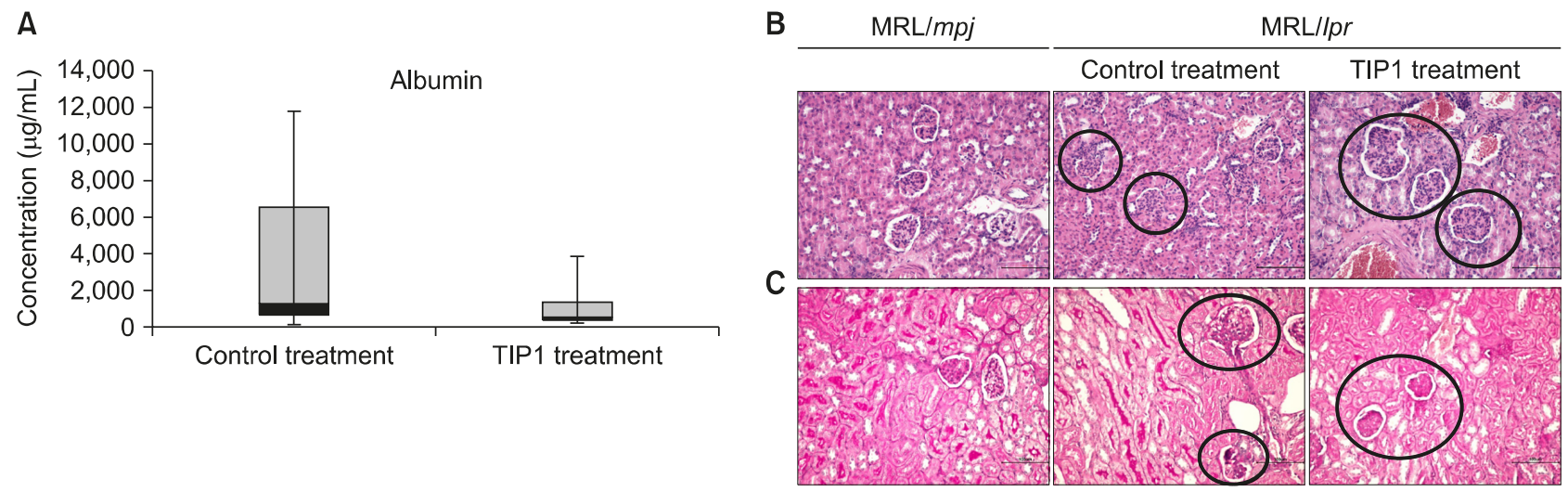

Figure 2. Toll-like receptor inhibitory peptide 1 (TIP1) treatment improved inflammation of the kidneys in lupus nephritis in MRL/lpr mice. (A) Urine albumin analysis. The results of the TIP1 treatment group and control treatment group were compared using the Mann-Whitney U-test. (B) H\&E staining $(\times 200)$ and (C) PAS staining $(\times 200)$ in the kidney tissues of the MRL/mpj normal control group, MRL/lpr control treatment group, and MRL/lpr TIP1 treatment group. Representative micrographs obtained from $\mathrm{H} \& \mathrm{E}$ and PAS staining of kidneys show differences in the glomeruli and surrounding inflammatory cells in mice of each group. The shape of the glomerulus in the control treatment group was destroyed, but that was maintained in the TIP1 treatment group (circle). 
teins are excreted in urine [19]. The urinary concentration of albumin, known as an indicator of renal inflammation, was decreased in the mice in the TIP1 treatment group when compared to the mice in the control treatment group (Figure 2A). In this study, the kidneys of mice were analyzed by $\mathrm{H} \& \mathrm{E}$ staining and PAS staining, and the proliferation of vascular mesentery cells, infiltration of surrounding inflammatory cells, and the size and shape of glomeruli were observed. The shape of the glomerulus of the mice in the control treatment group was destroyed, and the glomerulus was infiltrated by inflammatory cells, resulting in an irregular shape. However, in the mice in the TIP1 treatment group, the overall structure and shape of the glomerulus was constantly maintained (black circles, Figure 2B and 2C). In addition, the histopathological nephritis index also decreased in the mice of the TIP1 treatment group (Supplementary Figure 1). Therefore, intravenous treatment with TIP1 can improve inflammation in the kidney of lupus-prone mice.

\section{Autoantibodies and inflammatory cytokines after TIP1 treatment}

ANAs, anti-dsDNA antibodies, and serum IFN- $\alpha$, which are indicators of the presence of lupus in mouse serum, were measured by ELISA. ANAs tended to decrease in the mice in the TIP1 treatment group when compared with the mice in the control treatment group, but the difference was not statistically significant (Figure 3A). In addition, there was no statistically significant difference in the

\section{A}


anti-dsDNA antibody levels (Figure 3B) between the mice in the two groups. However, serum IFN- $\alpha$ levels were significantly $(\mathrm{p}<0.05)$ lower in the mice in the TIP1 treatment group than in the mice in the control treatment group (Figure 3C).

\section{TLR7 and TLR9 downstream signaling pathways in major organs}

It is known that the expression of TLR7 and TLR9 increases inflammatory cytokine levels and is thus related to the onset and progression of SLE [14-16]. Therefore, in this study, the protein expression levels of MyD88, IRAK4, TRAF6, and IRF7, which are downstream molecules in the signaling pathways of TLR7 and TLR9, were analyzed. The expression of TLR7, MyD88, and IRAK4 in the spleen and MyD88, IRAK4, and IRF7 in the kidneys was significantly decreased in the mice in the TIP1 treatment group, compared with those in the mice in the control treatment group (Figure 4A and 4B). Analysis of the mRNA of TLR7 and TLR9 in major tissues showed no significant difference between the TIP1 treatment and control treatment in the spleen and kidney (Figure 5A and 5B), but the expression of MyD88, IRAK4, TRAF6, IRF7, and IFN- $\alpha$ was significantly reduced in the lymph nodes of the mice in the TIP1 treatment group compared with that in the mice in the control treatment group (Figure 5C). From these results, it was concluded that the expression of TLR7 and several downstream signaling molecules was significantly decreased in the spleen, kidney,

B

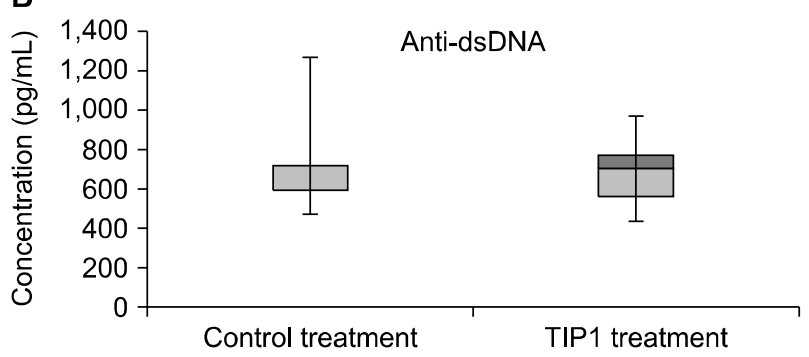

Figure 3. Serological and inflammatory markers after treatment of MRL/lpr mice with Toll-like receptor inhibitory peptide 1 (TIP1). (A) Antinuclear antibody (ANA), (B) anti-ds DNA antibody, and (C) interferon-alpha (IFN- $\alpha$ ). The results of the TIP1 treatment group and control treatment group were compared using the Mann-Whitney U-test. ${ }^{*} \mathrm{p}<0.05$. 
A
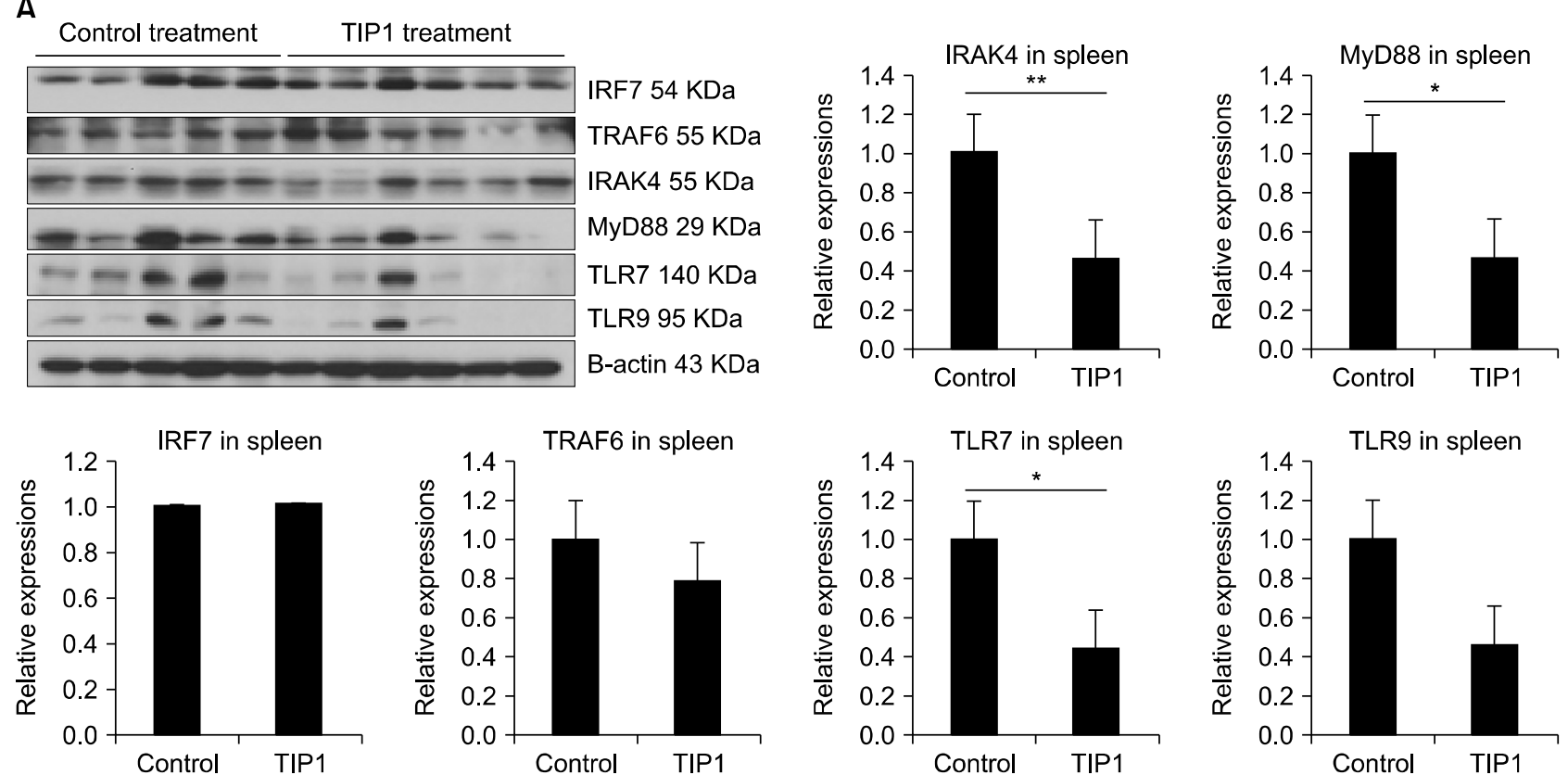

B


Figure 4. Toll-like receptor (TLR) inhibitory peptide (TIP) 1 blocks the TLR 7/9 downstream signaling pathway in major organs in mice. Western blot analysis of the expression levels the TLR 7/9 signaling pathway molecules in (A) spleen and (B) kidney of MRL/lpr mice. The bar graph shows the band intensity presented as the average value of the target protein to $\beta$-actin ratio $\left(^{*} \mathrm{p}<\right.$ $0.05, * * \mathrm{p}<0.01)$.

and lymph nodes of mice owing to TIP1 treatment.

\section{DISCUSSION}

The incidence of autoimmune diseases and thus the demand for understanding immune tolerance and activation is increasing globally. SLE, one of the most well-known autoimmune diseases, is known for its adverse effects on many different organs and tissues [20]. In addition, SLE is characterized by the production of autoantibodies against nuclear antigens, which are deposited in tissues to form immune complexes, causing inflammation [21,22].

Several studies have consistently been conducted to es- 
A
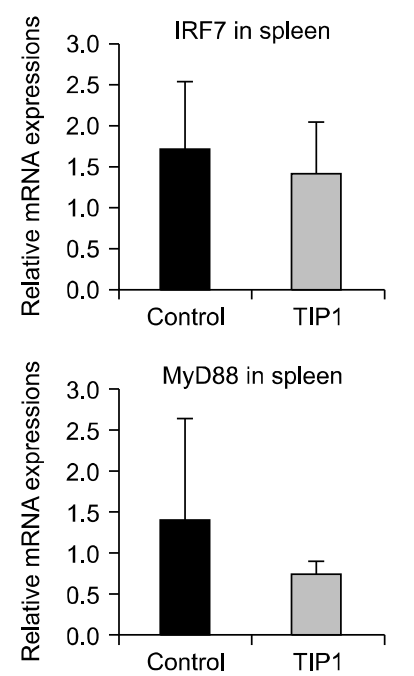

B
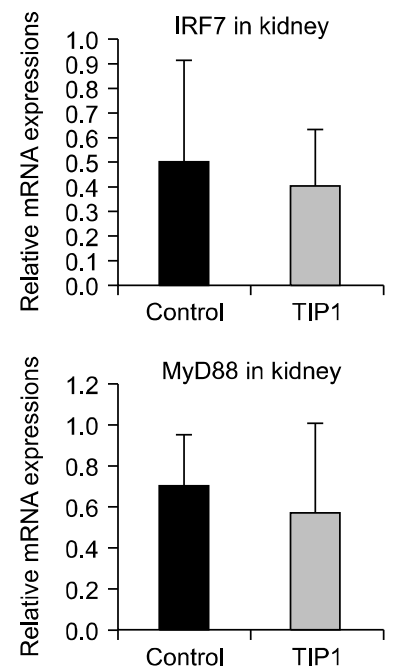

C
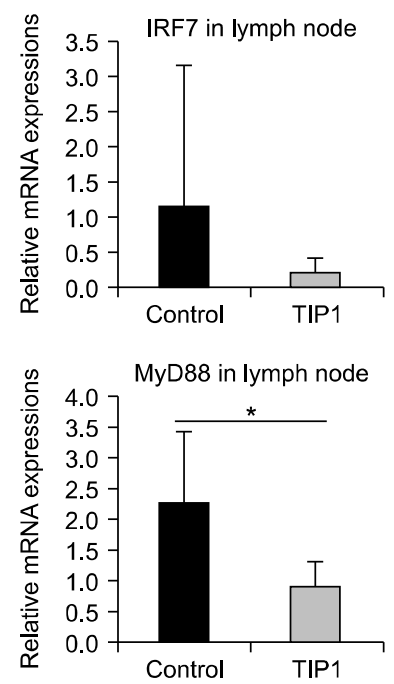

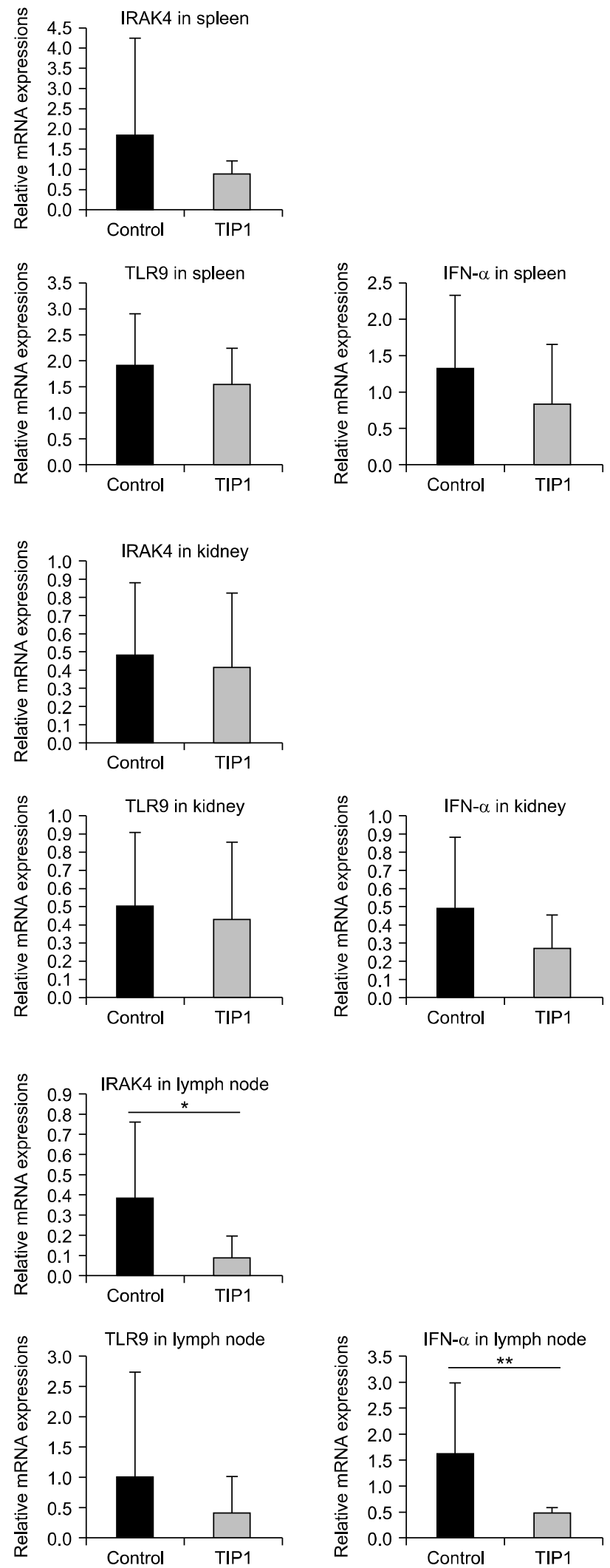

Figure 5. The mRNA expression level of proteins of the Toll-like receptor (TLR) 7/9 signaling pathway were analyzed using real-time polymerase chain reaction analysis in mouse (A) spleen, (B) kidney, and (C) lymph node. Using the Mann-Whitney U-test in the analysis, $p$-values less than 0.05 or 0.01 were considered statistically significant $\left({ }^{*} p<0.05, * * p<0.01\right)$. 
tablish a suitable treatment for SLE; however, there are considerable limitations to subjecting any of these treatments to clinical trials, although their effects were successfully demonstrated in animal testing. Nevertheless, several researchers are attempting to develop a more effective and safer treatment for SLE.

To date, many in vitro studies have provided evidence supporting the role of TLRs in the pathogenesis of SLE. TLRs are known to play an important role in the early detection of pathogen-associated molecular patterns and the subsequent activation of adaptive immune responses. Particularly, the recognition of nucleic acid motifs by TLRs is reported to induce disease progression after activation of antinuclear B cells and formation of immune complexes [23]. Many studies have reported the association of SLE with TLR7 and TLR9 [24-26]. Further, it was identified that the activation of TLRs leads to the production of inflammatory cytokines [14-16]. Therefore, TLRs can play an important role the pathogenesis of SLE [27]. Based on several prior studies, we decided that TLRs would be the most suitable target for the treatment of SLE. SLE results in inflammation of several organs and tissues and eventually causes damage. Thus, we investigated whether inflammatory cytokines decreased and SLE symptoms could be relieved when TIP1 is applied to inhibit TLR signaling in a lupus-prone animal model (MRL/lpr).

$\mathrm{MRL} / \mathrm{lpr}$ mice are representative animal models that present many organ pathologies and features of SLE [28,29]. According to existing studies using MRL/lpr models, the first SLE symptoms occurred at 14 weeks of age, and skin lesions and enlargement of the spleen and lymph nodes were observed $[29,30]$. In our study, however, skin lesions were observed only on the faces of the mice in each group, with no significant difference between the lesions of the mice in the TIP1 treatment and control treatment groups. In addition, the weight of the spleen tended to decrease to a small extent with TIP1 treatment, but the length of the spleen or kidney weight did not decrease significantly.

In lupus nephritis, glomeruli are damaged by kidney inflammation, and anti-dsDNA associated with the formation of immune complexes is detected [31-33]. In addition, exacerbating lupus nephritis results in higher urine albumin levels. Therefore, anti-dsDNA antibodies and urinary albumin can be indicators of lupus nephritis. In our study, experiments were conducted using various indicators of SLE to ascertain whether TIP1 treatment re- duced symptoms. As a result of TIP1 treatment, anti-dsDNA levels did not decrease, whereas urinary levels of albumin decreased, although not significantly, in comparison with the mice in the control treatment group.

Several studies on murine lupus models have suggested the role of TLR signaling in the pathogenesis of lupus nephritis [34]. Excessive production of TLR7 and TLR9 is commonly observed with the progression of lupus nephritis [35]. Recently reported findings have shown a correlation between TLR7 and TLR9 overexpression and clinical pathological indicators, supporting the role of mediators in the pathogenesis of lupus nephritis [29]. Similarly, in our study, the levels of TLR7, TLR9, and downstream signaling proteins MyD88, IRAK4, TRAF6, and IRF7 were reduced in TIP1-treated mice. These results indicate that when TIP1 binds to the BB loop region of the TIR domain, it blocks the TLR downstream signaling cascade through the MyD88 or TRIF adapter. Additionally, H\&E and PAS staining showed that the glomeruli of the mice in the TIP1 treatment group retained their shape, and the proliferation of cells was limited, which explains the improvement in kidney inflammation.

\section{CONCLUSION}

In this study, a representative SLE animal model (MRL/lpr) was used to determine whether SLE symptoms and signs reduced with TIP1 treatment. Treatment with TIP1 ameliorated lupus nephritis by reducing the concentration of urine albumin, retaining glomerular shape, and controlling the proliferation of mesenteric cells. In addition, TLR7, TLR9, and downstream signaling proteins decreased significantly in the lymph node, spleen, and kidney of the mice treated with TIP1. Further, IFN- $\alpha$, an inflammatory cytokine, was also significantly decreased. Therefore, TIP1 reduced the signs and symptoms of SLE by inhibiting TLR7, TLR9, and downstream signaling proteins. TIP1 could be a potential therapeutic agent for treating SLE.

\section{ACKNOWLEDGMENTS}

This research was supported by a grant from the Korea Health Technology R\&D Project through the Korea Health Industry Development Institute (KHIDI), funded by the Ministry of Health \& Welfare, Republic of Korea (grant number: HI16C0992). 


\section{CONFLICT OF INTEREST}

No potential conflict of interest relevant to this article was reported.

\section{AUTHOR CONTRIBUTIONS}

Conception and design of study: C.-H.S., S.C., W.Y.B. Acquisition of data: W.-Y.B., S.-M.L., S.-W.L., I.-O.S. Analysis and/or interpretation of data: C.-H.S., W.-Y.B., S.-M.L. Drafting the manuscript: W.-Y.B., S.-M.L. Revising the manuscript critically for important intellectual content: C.-H.S. All authors have read and approved the final version of the manuscript.

\section{SUPPLEMENTARY DATA}

Supplementary data can be found with this article online at https://doi.org/10.4078/jrd.2021.28.3.133.

\section{REFERENCES}

1. Tsokos GC. Systemic lupus erythematosus. N Engl J Med 2011;365:2110-21.

2. Klippel JH. Systemic lupus erythematosus: demographics, prognosis, and outcome. J Rheumatol Suppl 1997;48:67-71.

3. Petri M. Long-term outcomes in lupus. Am J Manag Care 2001;7(16 Suppl):S480-5.

4. Toblli JE, Trigo M, Mendoza G, Fellner JP, Genaro O, Houssay R, et al. [Hematologic manifestations in systemic lupus erythematosus. Experience collected from 150 cases]. Rev Clin Esp 1983;169:257-61. Spanish.

5. Kamen DL. Environmental influences on systemic lupus erythematosus expression. Rheum Dis Clin North Am 2014; 40:401-12, vii.

6. Kalok A, Abdul Cader R, Indirayani I, Abdul Karim AK, Shah SA, Mohamed Ismail NA, et al. Pregnancy outcomes in systemic lupus erythematosus (SLE) women. Horm Mol Biol Clin Investig 2019 Sep 25 [Epub]. DOI:10.1515/ hmbci-2019-0007.

7. Wincup C, McDonnell TCR, Rahman A. Menorrhagia: an underappreciated problem in pre-menopausal women with systemic lupus erythematosus. Lupus 2019;28:916-7.

8. Tsokos GC, Lo MS, Costa Reis P, Sullivan KE. New insights into the immunopathogenesis of systemic lupus erythematosus. Nat Rev Rheumatol 2016;12:716-30.

9. Leonard D, Eloranta ML, Hagberg N, Berggren O, Tandre K, Alm G, et al. Activated T cells enhance interferon- $\alpha$ production by plasmacytoid dendritic cells stimulated with RNA-containing immune complexes. Ann Rheum Dis 2016;75:1728-34.

10. Leadbetter EA, Rifkin IR, Hohlbaum AM, Beaudette BC, Shlomchik MJ, Marshak-Rothstein A. Chromatin-IgG com- plexes activate B cells by dual engagement of IgM and Toll-like receptors. Nature 2002;416:603-7.

11. Klonowska-Szymczyk A, Wolska A, Robak T, Cebula-Obrzut B, Smolewski P, Robak E. Expression of toll-like receptors 3,7 , and 9 in peripheral blood mononuclear cells from patients with systemic lupus erythematosus. Mediators Inflamm 2014;2014:381418.

12. Celhar T, Magalhães R, Fairhurst AM. TLR7 and TLR9 in SLE: when sensing self goes wrong. Immunol Res 2012;53: 58-77.

13. Christensen SR, Kashgarian M, Alexopoulou L, Flavell RA, Akira S, Shlomchik MJ. Toll-like receptor 9 controls anti-DNA autoantibody production in murine lupus. J Exp Med 2005;202:321-31.

14. Chauhan SK, Singh VV, Rai R, Rai M, Rai G. Distinct autoantibody profiles in systemic lupus erythematosus patients are selectively associated with TLR7 and TLR9 upregulation. J Clin Immunol 2013;33:954-64.

15. Akira S, Uematsu S, Takeuchi O. Pathogen recognition and innate immunity. Cell 2006;124:783-801.

16. Guiducci C, Gong M, Xu Z, Gill M, Chaussabel D, Meeker T, et al. TLR recognition of self nucleic acids hampers glucocorticoid activity in lupus. Nature 2010;465:937-41.

17. Kwon HK, Patra MC, Shin HJ, Gui X, Achek A, Panneerselvam $S$, et al. A cell-penetrating peptide blocks Toll-like receptor-mediated downstream signaling and ameliorates autoimmune and inflammatory diseases in mice. Exp Mol Med 2019;51:1-19.

18. Watson ML, Rao JK, Gilkeson GS, Ruiz P, Eicher EM, Pisetsky DS, et al. Genetic analysis of MRL-lpr mice: relationship of the Fas apoptosis gene to disease manifestations and renal disease-modifying loci. J Exp Med 1992;176: 1645-56.

19. Chen D, Hu W. Lupus podocytopathy: a distinct entity of lupus nephritis. J Nephrol 2018;31:629-34.

20. Celhar T, Lu HK, Benso L, Rakhilina L, Lee HY, Tripathi S, et al. TLR7 protein expression in mild and severe lupus-prone models is regulated in a leukocyte, genetic, and IRAK4 dependent manner. Front Immunol 2019;10:1546.

21. Suurmond J, Diamond B. Autoantibodies in systemic autoimmune diseases: specificity and pathogenicity. J Clin Invest 2015;125:2194-202.

22. Moulton VR, Suarez-Fueyo A, Meidan E, Li H, Mizui M, Tsokos GC. Pathogenesis of human systemic lupus erythematosus: a cellular perspective. Trends Mol Med 2017;23: 615-35.

23. Marshak-Rothstein A. Toll-like receptors in systemic autoimmune disease. Nat Rev Immunol 2006;6:823-35.

24. Nickerson KM, Christensen SR, Shupe J, Kashgarian M, Kim D, Elkon K, et al. TLR9 regulates TLR7- and MyD88dependent autoantibody production and disease in a murine model of lupus. J Immunol 2010;184:1840-8.

25. Celhar T, Fairhurst AM. Toll-like receptors in systemic lupus erythematosus: potential for personalized treatment. Front Pharmacol 2014;5:265.

26. Christensen SR, Shupe J, Nickerson K, Kashgarian M, Flavell RA, Shlomchik MJ. Toll-like receptor 7 and TLR9 dictate autoantibody specificity and have opposing inflammatory and regulatory roles in a murine model of lupus. Immunity 2006;25:417-28.

27. Wu YW, Tang W, Zuo JP. Toll-like receptors: potential tar- 
gets for lupus treatment. Acta Pharmacol Sin 2015;36:1395-407.

28. Tilstra JS, Avery L, Menk AV, Gordon RA, Smita S, Kane LP, et al. Kidney-infiltrating $\mathrm{T}$ cells in murine lupus nephritis are metabolically and functionally exhausted. J Clin Invest 2018;128:4884-97.

29. Liu J, Karypis G, Hippen KL, Vegoe AL, Ruiz P, Gilkeson GS, et al. Genomic view of systemic autoimmunity in MRLlpr mice. Genes Immun 2006;7:156-68.

30. Kim YY, Park KT, Jang SY, Lee KH, Byun JY, Suh KH, et al. HM71224, a selective Bruton's tyrosine kinase inhibitor, attenuates the development of murine lupus. Arthritis Res Ther 2017;19:211.

31. Yung S, Chan TM. Autoantibodies and resident renal cells in the pathogenesis of lupus nephritis: getting to know the unknown. Clin Dev Immunol 2012;2012:139365.

32. Rekvig OP. The dsDNA, anti-dsDNA antibody, and lupus nephritis: what we agree on, what must be done, and what the best strategy forward could be. Front Immunol 2019;10: 1104.

33. Goilav B, Putterman C. The role of anti-DNA antibodies in the development of lupus nephritis: a complementary, or alternative, viewpoint? Semin Nephrol 2015;35:439-43.

34. Devarapu SK, Anders HJ. Toll-like receptors in lupus nephritis. J Biomed Sci 2018;25:35.

35. Conti F, Spinelli FR, Truglia S, Miranda F, Alessandri C, Ceccarelli $\mathrm{F}$, et al. Kidney expression of Toll like receptors in lupus nephritis: quantification and clinicopathological correlations. Mediators Inflamm 2016;2016:7697592. 